\title{
Association between increase in vertical ground reaction force loading rate and pain level in women with patellofemoral pain after a patellofemoral joint loading protocol
}

\author{
Ronaldo Valdir Briani ${ }^{a}$, Marcella Ferraz Pazzinatto ${ }^{\mathrm{a}, \mathrm{b}}$, Marina Cabral Waiteman ${ }^{\mathrm{a}}$, \\ Danilo de Oliveira Silva ${ }^{\mathrm{a}, \mathrm{b}}$, Fábio Mícolis de Azevedo ${ }^{\mathrm{a}, *}$ \\ a Physical Therapy Department, School of Science and Technology, São Paulo State University (UNESP), Presidente Prudente, Brazil \\ ${ }^{\mathrm{b}}$ La Trobe University, School of Allied Health, La Trobe Sports and Exercise Medicine Research Centre (LASEM), Melbourne, Australia
}

\section{A R T I C L E I N F O}

\section{Article history:}

Received 11 October 2016

Received in revised form 11 September 2017

Accepted 12 March 2018

\section{Keywords:}

Ground reaction forces

Loading rates

Patellofemoral pain

Stair negotiation

\begin{abstract}
A B S T R A C T
Background: The etiology of patellofemoral pain (PFP) is thought to be the result of increased patellofemoral joint (PFJ) load and aberrant lower extremity mechanics, including altered vertical ground reaction forces (VGRF). However, few studies have investigated the association between an increase in pain and VGRF loading rates in the context of PFP. Thus, this study aimed to investigate the immediate effects of PFJ loading on pain and VGRF loading rate, and to see if there is a link between modification of both pain and VGRF loading rate during stair negotiation.

Methods: Thirty-four women with PFP underwent VGRF analysis during stair negotiation under two conditions: with (condition 2) and without (condition 1) being previously submitted to a PFJ loading protocol in order to or not to exacerbate their knee pain, respectively.

Results: The VGRF loading rates were significantly higher in condition 2 (Mean \pm standard deviation $(\mathrm{SD})=4.0 \pm 0.6 \mathrm{~N} / \mathrm{s}$ ) compared to condition 1 (Mean $\pm \mathrm{SD}=3.6 \pm 0.5 \mathrm{~N} / \mathrm{s}$ ) during stair ascent and during stair descent (Mean $\pm \mathrm{SD}$ : condition $1=6.3 \pm 1.1 \mathrm{~N} / \mathrm{s}$; condition $2=$ $7.0 \pm 1.4 \mathrm{~N} / \mathrm{s}$ ). In addition, VGRF loading rates were higher during stair descent compared to stair ascent in both conditions. There were significant correlations between the increase in pain and VGRF loading rate during both tasks.

Conclusion: There seemed to be an important relation between the increase in pain and VGRF loading rates in women with PFP. Based on these findings, interventions aimed at reducing VGRF loading rates are important in the context of PFP.
\end{abstract}

(c) 2018 Elsevier B.V. All rights reserved.

\section{Introduction}

Patellofemoral pain (PFP) is a common and costly musculoskeletal disorder that affects men, women and adolescents; women are 2.23 times more likely to develop PFP than men [1]. Previous studies have suggested that PFP may be a precursor to subsequent knee osteoarthritis in such populations [2,3]. Patellofemoral pain is characterized by pain around and behind the patella, and is aggravated by activities causing repetitive and high patellofemoral compressive forces such as stair ascent and descent, squatting and running [4,5]. Although this disorder accounts for $25-40 \%$ of all knee complaints in sports medicine [6], the underlying mechanisms remain unclear [7].

\footnotetext{
* Corresponding author at: Rua Roberto Simonsen, 305, Presidente Prudente, SP 19060-900, Brazil.

E-mail address: micolis@fct.unesp.br (F.M. de Azevedo).
} 
The etiology of PFP is thought to be the result of increased patellofemoral joint (PFJ) load and aberrant lower extremity mechanics [8], including altered vertical ground reaction forces (VGRF) [9-11]. The VGRF during heel strike in gait produce stress waves that are transmitted through the lower extremity up the kinetic chain [12]. These stress waves have been shown to be harmful when they are applied for short periods of time [13]. Since human tissue is viscoelastic, its loading response is timedependent (loading rates) and more prone to injury at higher loading rates [14]. Previous studies have investigated and found an association between high VGRF loading rates and joint degenerative process [13,14]. It is of interest that the general population with PFP has been found to exhibit higher VGRF loading rates compared to healthy individuals during stair ascent $[9,10]$. However, there are few of studies that have investigated the association between an increase in pain and VGRF loading rates in the context of PFP [9,11].

Higher VGRF loading rates in women with PFP compared to pain-free controls have been explained by altered knee kinematics [9]. Reduced knee flexion has been found in women with PFP and, although such a compensatory strategy is a logical approach to decreasing pain, it may hamper the lower extremity absorption mechanisms and, hence, may result in increased VGRF loading rates during negotiating stairs $[9,10]$. Therefore, there seems to be a direct relation between pain level and VGRF loading rates (i.e., the higher the pain, the higher the VGRF loading rates) [10]; however, such a novel approach has not previously been thoroughly investigated. In this line of reasoning, interventions aimed at reducing pain and VGRF loading rates may play an important role in reducing pain in the context of PFP [11,15].

One study has examined and found a relation between pain level and higher loading rates from the first peak of the VGRF in women with PFP compared to controls [10]. Nevertheless, the authors used a previous month of self-reported pain in the correlation model and, therefore, the immediate link between pain level and VGRF loading rates remains unknown. Given as such, there has not been an investigation into whether VGRF loading rates change immediately in the presence of pain, which could be an issue, as individuals with PFP tend to have intermittent pain characteristics [16]. Investigating whether individuals with PFP have different patterns of VGRF loading rates in the presence of pain would be important in the context of PFP. As a PFJ loading protocol has recently been shown to increase pain in women with PFP [16], its application could be used to overcome the aforementioned limitation.

Therefore, the aims of the current study were to investigate the immediate effects of PFJ loading on pain and VGRF loading rate, and to see if there is a link between the modification of both pain and VGRF loading rate during negotiating stairs. In addition, it aimed to determine the immediate effects of PFJ loading on the parameters that could influence and explain possible changes in the VGRF loading rate (i.e., VGRF first peak magnitude and stance time). It was hypothesized that there would be a mutual increase in pain level and VGRF loading rates, either in stair ascent or in stair descent, and that these two variables would be associated.

\section{Methods}

\subsection{Participants}

Thirty-four women with PFP were recruited via advertisements placed at the university, parks and gyms (demographics are presented in Table 1). Only women were included, due to the high prevalence of PFP in this population [1]. In addition, it was assumed that including both sexes could be seen as a confounding factor, as women have been reported to exhibit different movement patterns to men [17]. Based on calculations made in the sample power using Statistical Software for Social Sciences (SPSS) Version 18.0 (SPSS Inc. Chicago, IL, USA) with data from De Oliveira Silva et al. [10], a minimum sample size of 29 women was indicated to an expected $r=0.56$, with a statistical power of $80 \%$ and a significance level of five percent. Prior to data collection, all participants provided written informed consent and the experimental protocol was approved by the Institutional Review Board of the University of São Paulo State Human Ethics Committee (306.729).

Diagnosis of PFP was confirmed following consensus from two experienced clinicians ( $>5$ years' experience) and based on definitions used in previous studies [18-20]. The inclusion criteria were: (1) anterior knee pain during at least two of the following activities: prolonged sitting, squatting, kneeling, running, climbing stairs, and jumping; (2) pain during patellar palpation; (3) symptoms of insidious onset and duration of at least one month; (4) worst pain level in the previous month of at least three centimeters on a 10-cm visual analogue scale (VAS); and (5) two or more positive clinical signs in the following tests:

Table 1

Demographic data of the participants.

\begin{tabular}{ll}
\hline Parameters & \\
\hline Age, years & $22.6(2.1)$ \\
Height, $\mathrm{m}$ & $1.65(0.10)$ \\
Mass, $\mathrm{kg}$ & $70.2(7.7)$ \\
Bilateral symptoms, $\mathrm{n}$ & 10 \\
Dominance, $\mathrm{n}^{\text {Physical activity, MET } \cdot \mathrm{min} \cdot \mathrm{wk}^{-1}}$ & $21 \mathrm{right} / 13 \mathrm{left}$ \\
Bilateral/unilateral symptoms, $\mathrm{n}^{-1}$ & $4281.6(478.6)$ \\
\hline
\end{tabular}

Age, height, mass and physical activity are presented as mean and standard deviation. 
Clarke's sign, McConnell test, Noble compression and Waldron test. The participants were required to fulfill all five requirements to be included in the study. The presence of any of the following conditions were carefully screened as exclusion criteria: events of patellar subluxation or dislocation, lower limb inflammatory process, patellar tendon or meniscus tears, bursitis, ligament tears or the presence of neurological diseases. Those who had undergone knee surgery or received oral steroids, opiate treatment, acupuncture or physiotherapy during the preceding six months were excluded from this study.

\subsection{Instrumentation}

Data collection included lower limb kinetic evaluation of each participant's symptomatic limb (those with unilateral symptoms) or most symptomatic limb (in those with bilateral symptoms) during stair ascent and descent. The experimental design included a staircase with seven steps, each step being $28 \mathrm{~cm}$ deep and $18 \mathrm{~cm}$ high, with a walkway of two meters in front of and at the top of it [7]. A force plate (AMTI, OR6, Watertown, MA, USA) was positioned in the center of the fourth step and used to obtain VGRF data at a sampling frequency of $2000 \mathrm{~Hz}$. To ensure a natural stair ascent and descent pattern, participants were unaware of the force plate, which was covered by a rubberized fabric, making it impossible to distinguish the force plate from the other steps.

Subjects' pain was assessed using a 10-cm VAS. The extreme left side of the VAS stated no pain, whereas the extreme right side stated worst pain imaginable. Subjects drew a perpendicular line on the scale at the position that most likely described their pain at that moment. This scale has been validated and it is reliable for assessing women with PFP [21].

\subsection{Procedure}

The experimental design of this study was performed on four separate days (two days of condition 1 and two days of condition 2, in randomized order), with an interval of five days between data collection, in order to allow for reliability analysis and to not generate influences between protocols. Computer randomization software was used for each participant to generate a random order of four numbers (being numbers 1 and 2 for the first and second days of condition 1, 3 and 4 for the first and second days of condition 2); therefore, either the conditions or the days were randomized.

In condition 1, as soon as they arrived at the laboratory all participants were asked to rate their knee pain on a VAS, indicating the amount of pain they were feeling at that moment. Subsequently, each participant was asked to perform five repetitions of stair ascent and descent (total 10). In condition 2, before participants rated their knee pain they were submitted to a PFJ loading protocol, in order to exacerbate their symptoms [16]. The PFJ loading protocol involved a staircase with seven steps, where the participants performed 15 repetitions of stair negotiation with 35\% external load of the subject's body mass (carried in a backpack) [16]. A metronome was used at 96 steps/min to standardize the rhythm of stair negotiation used during the PFJ loading protocol [16]. Briani et al. tested and found that a PFJ loading protocol did not generate neuromuscular fatigue in women with PFP [22]. Afterwards, the participants rated their knee pain and performed five repetitions of stair ascent and descent again (total 10). Participants were advised to avoid taking medications during and between the assessments, as well as changing common habits and physical activity levels.

\subsection{Data analysis}

Each trial was filtered with a fourth-order Butterworth low-pass filter with a cutoff frequency of $10 \mathrm{~Hz}$ [23]. The VGRF parameter of interest was average loading rate. The average of the VGRF loading rate was chosen because it is the method of calculating the loading rate most commonly found in PFP studies [9-11,15]. The VGRF loading rate was calculated as between 20 and $80 \%$ of the period between foot strike and first peak of VGRF, and the loading rate calculation was the total change in VGRF divided by the total change in time over this period [12]. In addition, the first peak of the VGRF and the stance time were obtained. The mean of the five trials was used for statistical analysis.

\subsection{Reliability}

In order to guarantee consistency of the findings, the reliability of the variable of interest was analyzed in both conditions, with and without the PFJ loading protocol, considering the entire sample. For a relative measure of reliability, the intraclass correlation coefficient (ICC) $(2, \mathrm{k})$ model (confidence intervals) was used and the standard error of measurement (SEM) was used to express the precision of the measurement in absolute values. In order to clarify interpretation of the SEM, the values were also expressed as a percentage of the mean. For condition 1, the ICC and SEM values during stair descent were $0.86(0.68 ; 0.93)$ and 0.28 (7\%; and during stair ascent they were $0.93(0.86 ; 0.97)$ and 0.31 (8\%), respectively. Likewise, for condition 2, the ICC and SEM values during stair descent were $0.88(0.81 ; 0.90)$ and 0.31 (7.8\%); and during stair ascent they were $0.78(0.76 ; 0.82)$ and $0.34(9.7 \%)$, respectively. Therefore, in both conditions, VGRF loading rate showed satisfactory reliability. 


\subsection{Statistical analysis}

All analyses were performed using Statistical Package for the Social Sciences software program (version 18.0, SPSS, INC., Chicago, IL) with an a priori level of significance of 0.05 . Normality and variance homogeneity of data were tested using the Shapiro-Wilk and Levene tests, respectively.

Due to adequate values of reliability, data used for subsequent analyses were randomly selected from one day for each condition. Dependent $t$-tests were used to compare self-reported pain levels between both conditions. Effect sizes were calculated and interpreted according to Cohen [24]. In addition, a $2 \times 2$ analysis of variance (ANOVA) was used to compare VGRF loading rate, first peak and stance time between both conditions during stair ascent and descent. The Bonferroni post hoc test was performed for multiple pairwise comparisons where appropriate. The data reported from ANOVA were the $\mathrm{F}$ values (with degrees of freedom), $P$-values and the eta squared $(\eta 2)$. A Pearson product-moment correlation matrix was used to examine the relationship between increase in VGRF loading rate and pain level. For this purpose, the score change from condition 1 to condition 2 was used in the correlation model (i.e., condition 1-condition $2=$ score change). Therefore, positive values indicated higher values in condition 1, and negative values indicated higher values in condition 2.

\section{Results}

Self-reported pain level was significantly higher in condition $2(4.1 \pm 1.9 \mathrm{~cm})$ compared to condition $1(2.9 \pm 2.2 \mathrm{~cm})$ with a large effect size $\left(\mathrm{t}_{(33)}=-3.296, P=0.002, d=-4.72\right)$ (Figure 1). The results revealed significant effects of task $\left(\mathrm{F}_{(1,33)}=\right.$ $140.797, P=0.000, \eta 2=0.68)$ and effects of time were found for VGRF loading rates $\left(F_{(1,33)}=26.464, P=0.000, \eta 2=0.28\right)$, but not interaction $\left(\mathrm{F}_{(2,66)}=2.340, P=0.131, \eta 2=0.03\right)$. The VGRF loading rates were significantly higher in condition 2 $(4.0 \pm 0.6 \mathrm{~N} / \mathrm{s})$ compared to condition $1(3.6 \pm 0.5 \mathrm{~N} / \mathrm{s})$ during stair ascent $(P=0.005)$ and during stair descent (condition $1=6.3 \pm 1.1 \mathrm{~N} / \mathrm{s}$; condition $2=7.0 \pm 1.4 \mathrm{~N} / \mathrm{s})(P=0.01)$ (Figure 2). The VGRF loading rates were also higher during stair descent compared to stair ascent in both conditions.

Significant effects of task $\left(F_{(1,33)}=8.431, P=0.002, \eta 2=0.16\right)$ but no effects of time $\left(F_{(1,33)}=1.560, P=0.217, \eta 2=\right.$ 0.02 ) and interaction $\left(\mathrm{F}_{(2,66)}=0.051, P=0.822, \eta 2=0.01\right)$ were found for the first peak of the VRGF (Table 2$)$. First peak values were significantly higher during stair descent in both conditions (condition $1=13.0 \pm 1.25$; condition $2=13.2 \pm 0.9$ ) than during stair ascent (condition $1=10.0 \pm 0.4$; condition $2=10.1 \pm 0.7$ ), but did not change from condition 1 to 2 . In addition, significant effects of task $\left(F_{(1,33)}=5.851, P=0.037, \eta 2=0.10\right)$ and effects of time were found for stance time $\left(F_{(1,33)}=4.078\right.$, $P=0.048, \eta 2=0.08)$, but not interaction $\left(\mathrm{F}_{(2,66)}=1.963, P=0.390, \eta 2=0.02\right)$. Stance time was significantly lower during stair descent in both conditions (condition $1=0.75 \pm 0.08$; condition $2=0.68 \pm 0.06$ ) than during stair ascent (condition $1=0.85 \pm 0.08$; condition $2=0.79 \pm 0.10$ ). Also, in both tasks, the stance time was lower in condition 2 than in condition 1 .

There were significantly positive correlations between the increase in pain and VGRF loading rate from condition 1 to condition 2 during stair ascent $(P=0.012, r=0.46)$ (Figure 3 ) and during stair descent $(P=0.000, r=0.76)$ (Figure 4), although the correlation during stair ascent was low and during stair descent was high.

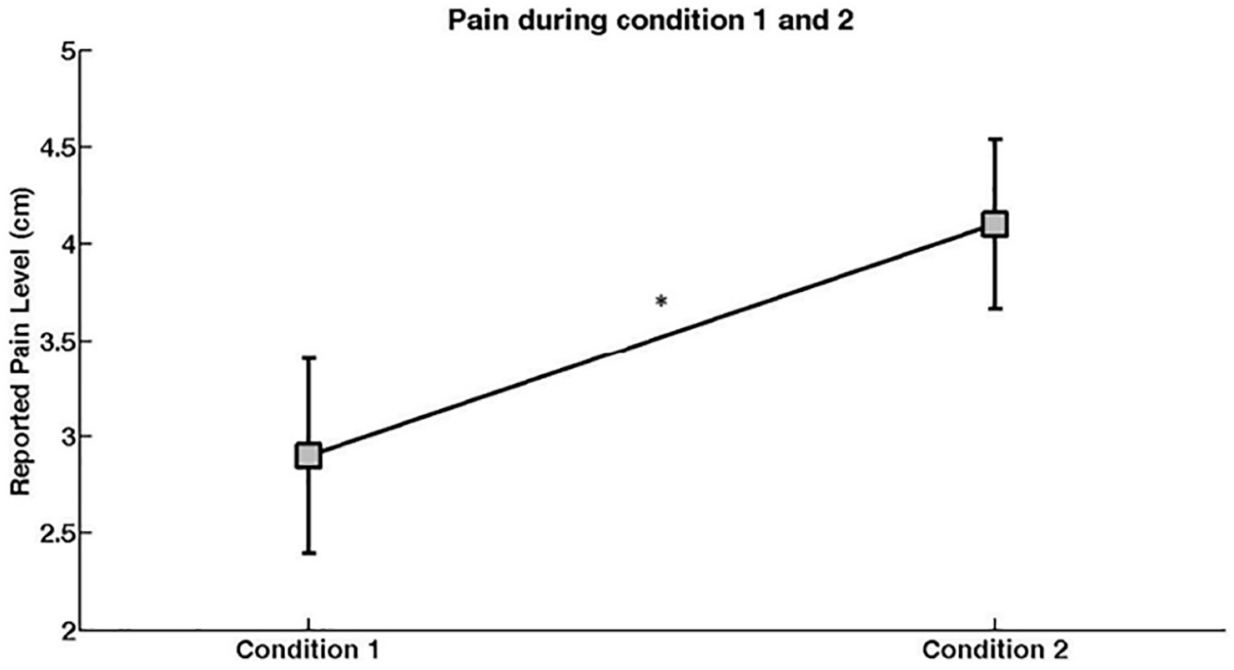

Figure 1. Self-reported pain of women with patellofemoral pain during both experimental conditions. Condition $1=$ pain without being submitted to the patellofemoral joint loading protocol. Condition $2=$ pain after being submitted to the patellofemoral joint loading protocol. *Statistically significant $(P<0.05)$ difference. Data are presented as mean and standard deviations. 


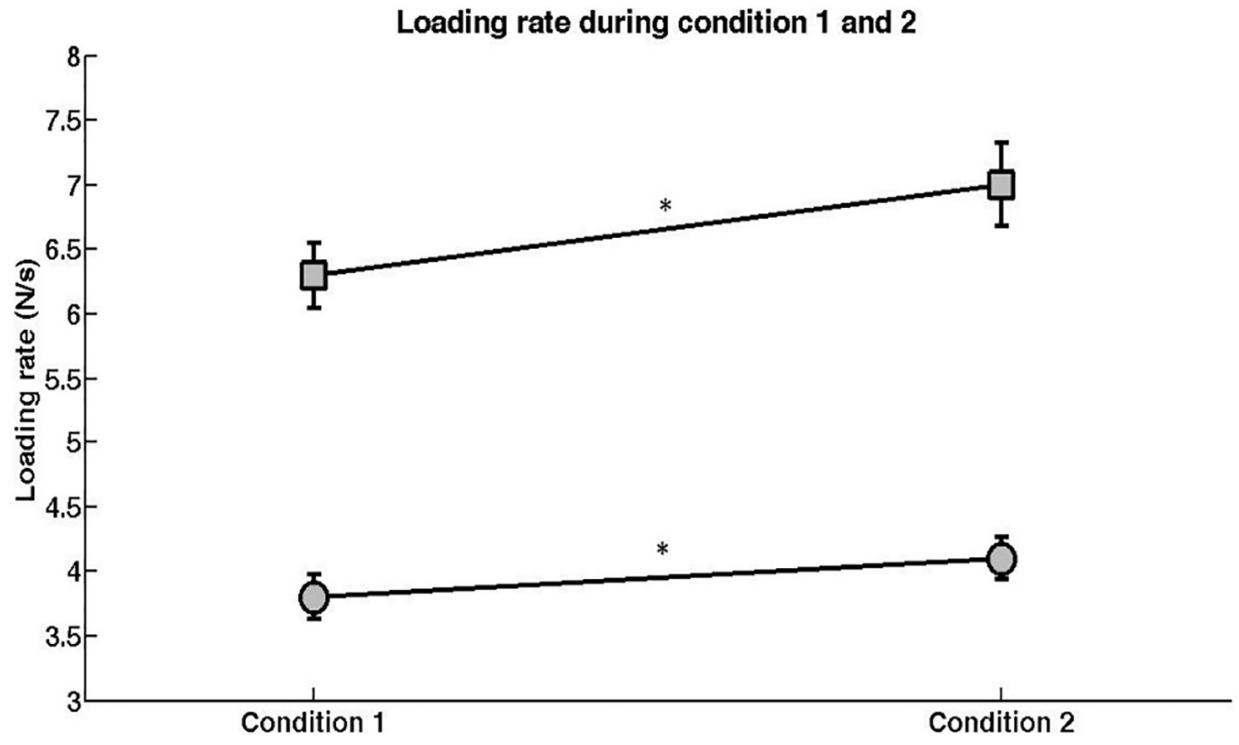

Figure 2. Vertical ground reaction force loading rates of women with patellofemoral pain during both experimental conditions. VGRF $=$ vertical ground reaction force; PFJ = patellofemoral joint. Condition $1=$ VRGF loading rates without being submitted to the PFJ loading protocol Condition $2=$ VGRF loading rates after being submitted to the PFJ loading protocol. *Statistically significant $(P<0.05)$ difference. Circles, represent VRGF loading rates during stair ascent. Squares, represent VGRF loading rates during stair descent. Data are presented as mean and standard deviations.

\section{Discussion}

After the PFJ loading protocol, knee pain was significantly increased in women with PFP, which was accompanied by an increase in VGRF loading rate and a decrease in stance time during stair ascent and descent (first peak values remained without difference). Higher values of VGRF loading rates and first peak and lower stance time were found during stair descent compared to stair ascent during both conditions. Positive correlations were also found between the increase in both pain and VGRF loading rate during stair ascent and descent.

These findings are supported by Chueng et al. [11] and Esculier et al. [15] who found that women with PFP who had a decrease in pain level after rehabilitation also had a reduction in VGRF loading rate during running. Interestingly, Esculier et al. found that women with PFP who did not report improvements in their symptoms were unable to reduce the VGRF loading rate [15]. In addition, a significant correlation was found by Esculier et al. between the reduction in instantaneous VGRF loading rate and improvements in symptoms and functional status of women with PFP [15]. Although there is a difference in the methods of calculating the loading rate between Esculier's study and the present study (instantaneous and average loading rate, respectively), both methods are similar and widely used for studying lower extremity loading [25,26]. Therefore, an important relation between increased VGRF loading rates and PFP seems to exist, which should be the focus of target interventions.

Biomechanically, the link between VGRF loading rate and pain is plausible. It is likely that high VGRF loading rates may be linked to increased PFJ loading rates [11,27]. Repetitive high-impact forces may compromise lower extremity articular structures and contribute to overuse injuries such as PFP [28]. It has been suggested that PFP may include repetitive and abnormal load distribution around the synovium, infrapatellar fat pad, retinacula and subchondral bone [28]. Dye suggested that altered load shifting produces changes in the mechanical and chemical environments of the knee [29]. These changes may trigger nociceptive activity that leads to PFP [29]. The findings of de Oliveira Silva et al. and the current ones support this theory, since self-reported

Table 2

First peak of the ground reaction force and stance time during stair ascent and descent.

\begin{tabular}{llc}
\hline Parameters & & \\
\hline Condition 1 & Stair ascent & Stair descent \\
First peak (BW) & $10.0(0.47)^{\mathrm{a}}$ & $13.0(1.25)^{\mathrm{a}}$ \\
Stance time (seconds) & $0.85(0.08)^{\mathrm{a}, \mathrm{b}}$ & $0.75(0.08)^{\mathrm{a}, \mathrm{b}}$ \\
Condition 2 & & $13.2(0.95)^{\mathrm{a}}$ \\
First peak (BW) & $10.1(0.79)^{\mathrm{a}}$ & $0.68(0.06)^{\mathrm{a}, \mathrm{b}}$ \\
Stance time (seconds) & $0.79(0.10)^{\mathrm{a}, \mathrm{b}}$ & \\
\hline
\end{tabular}

BW = Body weight.

a Represents differences between tasks (stair ascents vs stair descent).

b Represents differences between conditions (condition 1 vs condition 2). 


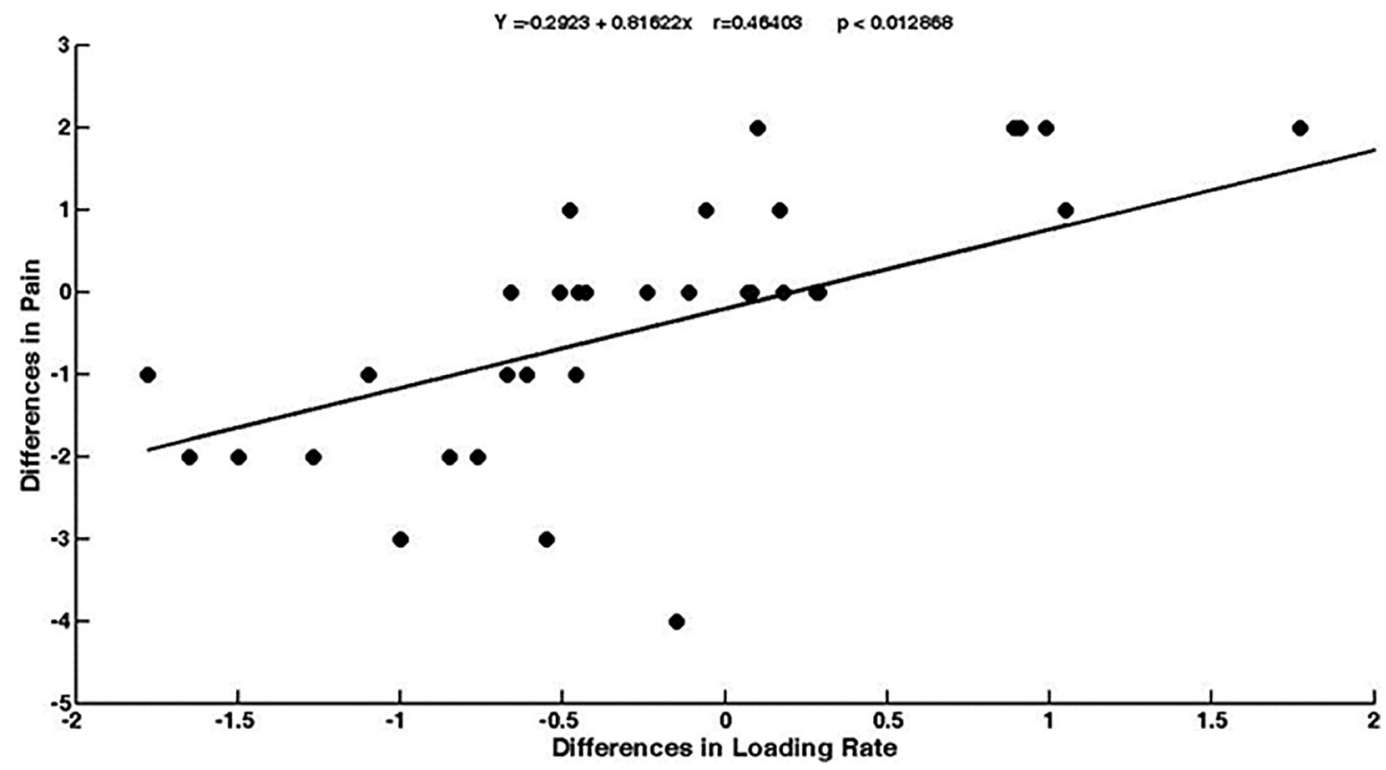

Figure 3. Pearson correlation coefficient between the increase in pain levels and in vertical ground reaction force loading rates during stair ascent. Note: The data of three subjects are superimposed by others.

pain was positively correlated with VGRF loading rate, which indicates that higher levels of pain are correlated with higher values of VGRF loading rate [10]. Nonetheless, it remains unclear whether VGRF loading rates contribute to PFP as a cause or an effect.

According to the current findings, the increase in VGRF loading rate (from condition 1 to 2 ) occurred mostly due to a decrease in stance time (shorter loading time). This finding corroborates with Cook et al. who stated that walking speed is a major determinant of VGRF characteristics and suggested that a patient might benefit from instructions to walk at slower speeds [30]. On the other hand, the increase in the VGRF loading rate in the current findings did not seem to be related to the first peak of the VGRF, since this parameter did not change from condition 1 to 2 . In terms of the differences between tasks, the higher values of VGRF loading rate during stair descent compared to stair ascent seem to also be related to the shorter stance time. However, in this case, the magnitude of the first peak of the VGRF also contributed to the higher values of VGRF loading rate during stair descent, which explains the large differences between tasks. Additionally, as shown by Brechter and Powers [31], greater PFJ stress, lower knee flexion, muscular and mechanical demands were found in stair descent compared to stair ascent, which

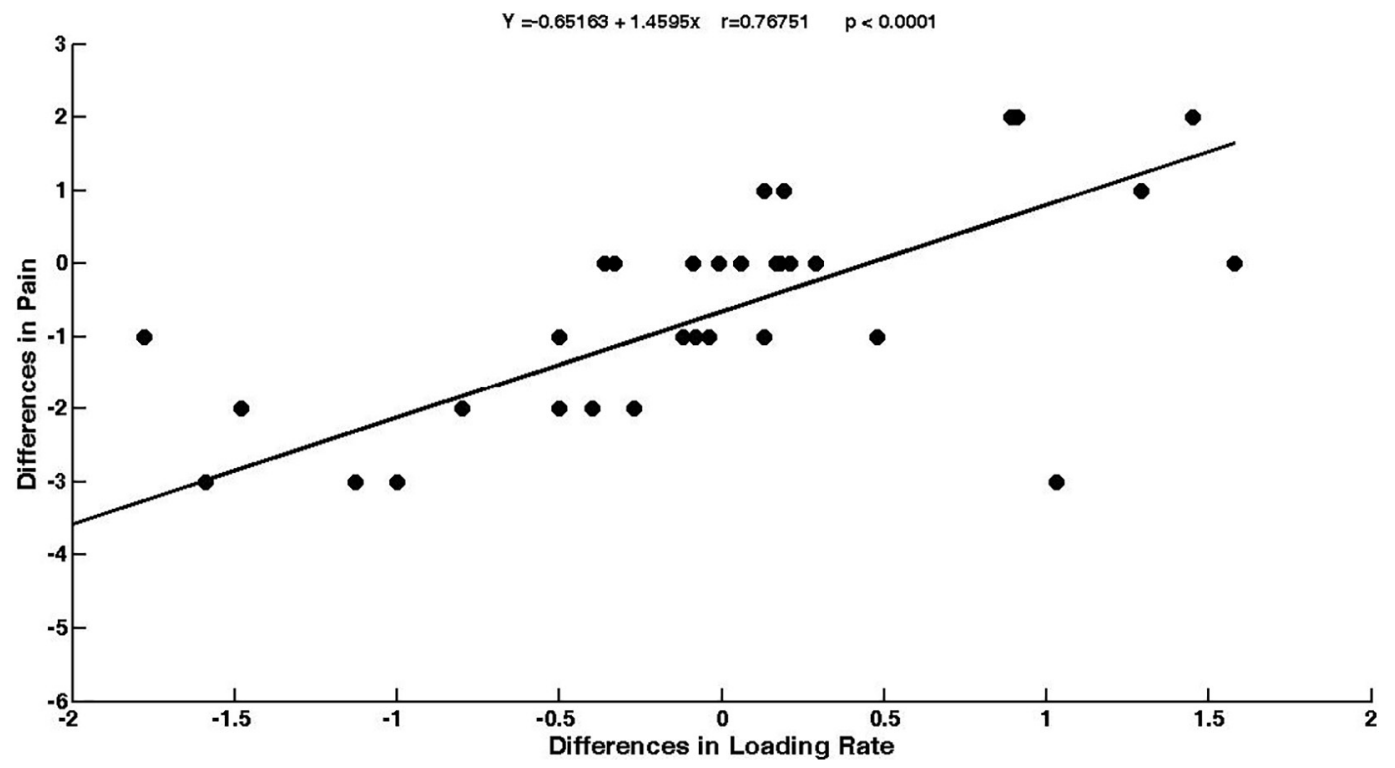

Figure 4. Pearson correlation coefficient between the increase in pain levels and in vertical ground reaction force loading rates during stair descent. Note: The data of two subjects are superimposed by others. 
may have also influenced the magnitude of the first peak and overall stance time of the VGRF, leading to increases in the VGRF loading rate.

\subsection{Clinical implications}

Rehabilitation programs aimed at gait retraining, using either visual [25] or verbal feedback [11,15], seem to be effective in reducing VGRF loading rates and symptoms of women with PFP. Simple, task-specific advice to "reduce noise" and actively modify the foot strike pattern to midfoot or forefoot strike during stair negotiation may cause significant improvements in pain, function and also reduce VGRF loading rates, as has been shown in running [11,15]. As stair negotiation is an activity performed constantly on a daily basis and, given the current results, performing it wrongly might be deleterious to the knee joint, studies should be conducted in order to investigate the effect of stair negotiation retraining on pain and biomechanics of individuals with PFP. Studies have shown that negotiating stairs is a vigorous activity that can lead to several health benefits; [32,33] therefore, learning to decrease VGRF loading rates during stair negotiation could allow increasing activity levels without increasing pain in women with PFP. In addition, studies have also suggested interventions to re-establish reduced knee flexion (i.e., strengthening, stretching, knee braces), due to its relation to increased VGRF loading rates [9,10]. Thus, based on the findings of the current and previous studies $[11,15,25]$, interventions aimed at reducing VGRF loading rates are important in the context of PFP.

\subsection{Limitations}

This study had some limitations that should be acknowledged. Firstly, the sample included only women. Although this subgroup was important to study, as they are individuals that are the most likely to suffer from PFP, the results may not be generalizable to the entire population of people with PFP. Secondly, due to the cross-sectional design, it was not possible to differentiate between cause and effect in relation to the altered VGRF loading rates and PFP. Thirdly, women with unilateral and bilateral knee symptoms were included and, although not critical because $15 \%$ of the sample had bilateral symptoms, pain in the non-tested limb may have played a role in the value of VGRF loading rate of the tested limb during stair descent. A decrease in the external knee extensor moment of the non-tested limb might influence the weight acceptance of the tested limb during stair descent. Lastly, because only stair ascent and descent were investigated, the findings may not be generalizable to other tasks. Further prospective research is needed to evaluate if increased VGRF loading rate is the cause or caused by PFP.

\section{Conclusion}

After performing a protocol designed to overload their PFJ, women with PFP presented increases in the self-reported pain and VGRF loading rates and decreases in stance time during stair ascent and descent. In addition, there was an association between the increase in VGRF loading rates and pain either in stair ascent or stair descent. These findings may indicate a deficit in the lower extremity absorption mechanisms of women with PFP, mainly in the presence of pain.

\section{Acknowledgments}

This work was developed in the Laboratory of Biomechanics and Motor Control at the Sao Paulo State University.

\section{Financial interest}

To São Paulo Research Foundation (FAPESP) for a grant (2014/24939-7) and a scholarship (2015/00406-2). The financial sponsors played no role in the design, execution, analysis and interpretation of data or writing of the study.

\section{References}

[1] Boling M, Padua D, Marshall S, Guskiewicz K, Pyne S, Beutler A. Gender differences in the incidence and prevalence of patellofemoral pain syndrome. Scand J Med Sci Sports 2010;20:725-30. https://doi.org/10.1111/j.1600-0838.2009.00996.x.

[2] Wyndow N, Collins N, Vicenzino B, Tucker K, Crossley K. Is there a biomechanical link between patellofemoral pain and osteoarthritis? A narrative review. Sports Med 2016. https://doi.org/10.1007/s40279-016-0545-6.

[3] Thomas MJ, Wood L, Selfe J, Peat G. Anterior knee pain in younger adults as a precursor to subsequent patellofemoral osteoarthritis: a systematic review. BMC Musculoskelet Disord 2010;11:201. https://doi.org/10.1186/1471-2474-11-201.

[4] De Oliveira Silva D, Briani RV, Pazzinatto MF, Ferrari D, Aragão FA, de Albuquerque CE, Alves N, de Azevedo FM. Reliability and differentiation capability of dynamic and static kinematic measurements of rearfoot eversion in patellofemoral pain. Clin Biomech 2015;30:144-8. https://doi.org/10.1016/j.clinbiomech. 2014.12.009.

[5] Crossley KM, Stefanik JJ, Selfe J, Collins NJ, Davis IS, Powers CM, McConnell J, Vicenzino B, Bazett-Jones DM, Esculier JF, Morrissey D, Callaghan MJ. Patellofemoral pain consensus statement from the 4th International Patellofemoral Pain Research Retreat, Manchester. Part 1: terminology, definitions, clinical examination, natural history, patellofemoral osteoarthritis and patient-reported outcome. Br J Sports Med 2016. https://doi.org/10.1136/bjsports-2016-096268.

[6] Fagan V, Delahunt E. Patellofemoral pain syndrome: a review on the associated neuromuscular deficits and current treatment options. Br J Sports Med 2008;42: 789-95. https://doi.org/10.1136/bjsm.2008.046623.

[7] De Oliveira Silva D, Magalhães FH, Pazzinatto MF, Briani RV, Ferreira AS, Aragão FA, de Azevedo FM. Contribution of altered hip, knee and foot kinematics to dynamic postural impairments in females with patellofemoral pain during stair ascent. Knee 2016;23:376-81. https://doi.org/10.1016/j.knee.2016.01.014.

[8] De Oliveira Silva D, Barton CJ, Pazzinatto MF, Briani RV, de Azevedo FM. Proximal mechanics during stair ascent are more discriminate of females with patellofemoral pain than distal mechanics. Clin Biomech 2016;35:56-61. https://doi.org/10.1016/j.clinbiomech.2016.04.009. 
[9] De Oliveira Silva D, Briani RV, Pazzinatto MF, Ferrari D, Aragão FA, de Azevedo FM. Reduced knee flexion is a possible cause of increased loading rates in individuals with patellofemoral pain. Clin Biomech 2015;30:971-5. https://doi.org/10.1016/j.clinbiomech.2015.06.021.

[10] De Oliveira Silva D, Briani R, Pazzinatto M, Ferrari D, Aragão F, De Azevedo F. Vertical ground reaction forces are associated with pain and self-reported functional status in recreational athletes with patellofemoral pain. J Appl Biomech 2015;31:409-14. https://doi.org/10.1123/jab.2015-0048.

[11] Chueng RT, Davis IS. Landing pattern modification to improve patellofemoral pain in runners: a case series. J Orthop Sports Phys Ther 2011;41:914-9. https://doi. org/10.2519/jospt.2011.3771.

[12] Collins JJ, Whittle MW. Impulsive forces during walking and their clinical implications. Clin Biomech 1989;4:179-87. https://doi.org/10.1016/0268-0033(89) 90023-5.

[13] Radin EL, Yang KH, Riegger C, Kish VL, O'Connor JJ. Relationship between lower limb dynamics and knee joint pain. J Orthop Res 1991;9:398-405. https://doi.org/ 10.1002/jor.1100090312.

[14] Ewers BJ, Jayaraman VM, Banglmaier RF, Haut RC. Rate of blunt impact loading affects changes in retropatellar cartilage and underlying bone in the rabbit patella. J Biomech 2002;35:747-55. https://doi.org/10.1016/S0021-9290(02)00019-2.

[15] Esculier JF, Bouyer LJ, Roy JS. The effects of a multimodal rehabilitation program on symptoms and ground-reaction forces in runners with patellofemoral pain syndrome. J Sport Rehabil 2016;25:23-30. https://oi.org/10.1123/jsr.2014-0245.

[16] Pazzinatto MF, de Oliveira Silva D, Barton CJ, Rathleff MS, Briani RV, Azevedo FM. Female adults with patellofemoral pain are characterized by widespread hyperalgesia, which is not affected immediately by patellofemoral joint loading. Pain Med 2016;17:1953-61. https://doi.org/10.1093/pm/pnw068

[17] Willy RW, Manal KT, Witvrouw E, Davis IS. Are mechanics different between male and female runners with patellofemoral pain? Med Sci Sports Exerc 2012;44: 2165-71. https://doi.org/10.1249/MSS.0b013e3182629215.

[18] Briani RV, de Oliveira Silva D, Pazzinatto MF, Ferreira AS, Ferrari D, de Azevedo FM. Delayed onset of electromyographic activity of the vastus medialis relative to the vastus lateralis may be related to physical activity levels in females with patellofemoral pain. J Electromyogr Kinesiol 2016;26:137-42. https://oi.org/10. 1016/j.jelekin.2015.10.012.

[19] De Oliveira Silva D, Briani RV, Pazzinatto MF, Gonçalves AV, Ferrari D, Aragão FA, de Azevedo FM. Q-angle static or dynamic measurements, which is the best choice for patellofemoral pain? Clin Biomech 2015;30:1083-7. https://doi.org/10.1016/j.clinbiomech.2015.09.002.

[20] De Oliveira Silva D, Magalhães FH, Faria NCS, Pazzinatto MF, Ferrari D, Pappas E, de Azevedo FM. Lower amplitude of H-reflex in females with patellofemoral pain thinking beyond proximal, local and distal factors. Arch Phys Med Rehabil 2016;97:1115-20. https://doi.org/10.1016/j.apmr.2015.12.017.

[21] Crossley KM, Bennell KL, Cowan SM, Green S. Analysis of outcome measures for persons with patellofemoral pain: which are reliable and valid? Arch Phys Med Rehabil 2004;85:815-22. https://doi.org/10.1016/S0003-9993(03)00613-0.

[22] Briani RV, Pazzinatto MF, Silva DO, Azevedo FM. Different pain responses to distinct levels of physical activity in women with patellofemoral pain. Braz J Phys Ther 2017;21:138-43. https://doi.org/10.1016/j.bjpt.2017.03.009.

[23] Briani RV, de Oliveira Silva D, Pazzinatto MF, de Albuquerque CE, Ferrari D, Aragão FA, de Azevedo FM. Comparison of frequency and time domain electromyography parameters in women with patellofemoral pain. Clin Biomech 2015;30:302-7. https://doi.org/10.1016/j.clinbiomech.2014.12.014.

[24] Cohen J. Statistical power analysis for the behavioral sciences. , 2New York: Routledge; 1988.

[25] Crowell HP, Davis IS. Gait retraining to reduce lower extremity loading in runners. Clin Biomech 2011;26:78-83. https://doi.org/10.1126/scisignal.2001449. Engineering.

[26] Cheung RTH, Rainbow MJ. Landing pattern and vertical loading rates during first attempt of barefoot running in habitual shod runners. Hum Mov Sci 2014;34 120-7. https://doi.org/10.1016/j.humov.2014.01.006.

[27] Lenhart RL, Thelen DG, Wille CM, Chumanov ES, Heiderscheit BC. Increasing running step rate reduces patellofemoral joint forces. Med Sci Sports Exerc 2014;46: 557-64. https://doi.org/10.1249/MSS.0b013e3182a78c3a.

[28] Harrison A, Ford K, Myer G, Hewett T. Sex differences in force attenuation: a clinical assessment of single-leg hop performance on a portable force plate. Br J Sports Med 2011;45:198-202. https://doi.org/10.1038/nature13314.A.

[29] Dye S. The pathophysiology of patellofemoral pain. Clin Orthop Relat Res 2005:100-10.

[30] Cook TM, Farrell KP, Carey IA, Gibbs JM, Wiger GE. Effects of restricted knee flexion and walking speed on the vertical ground reaction force during gait. J Orthop Sports Phys Ther 1997;25:236-44. https://doi.org/10.2519/jospt.1997.25.4.236.

[31] Brechter JH, Powers CM. Patellofemoral joint stress during stair ascent and descent in persons with and without patellofemoral pain. Gait Posture 2002;16: $115-23$.

[32] Teh KC, Aziz AR. Heart rate, oxygen uptake, and energy cost of ascending and descending the stairs. Med Sci Sports Exerc 2002;34:695-9. https://doi.org/10.1097/ 00005768-200204000-00021.

[33] Basset DR, Vachon JA, Kirkland AO, Howley ET, Duncan GE, Johnson KR. Energy cost of stair climbing and descending on the college alumnus questionnaire. Med Sci Sports Exerc 1997;29:1250-4. 